\title{
Supporting research as well as compliance
}

\author{
Jamie Lewis, BA, RLATG \& Judy Barnett, BS, RLATG, CMAR, \\ Protocol Advisors and Liaisons, Post-approval Monitoring Program, \\ Research Services, University of Texas Medical Branch, Galveston, TX
}

Ms. Lewis and Ms. Barnett talk about how their combined strengths contribute to the success of their PAM program.

Tell us about each of your career paths. How did you come to work as Protocol Advisors and Liaisons at the University of Texas Medical Branch?

We were at University of Texas Medical Branch (UTMB) for many years before the Post-approval Monitoring (PAM) program existed. Jamie was a basic science researcher for 21 years, studying epithelial transport pathways in USDA-covered species. Judy worked in many aspects of animal husbandry, including vivarium supervision, for 11 years. Both of these career paths stemmed from a love of animals, an interest in science, an educational background in biology, and a modicum of serendipity!

Jamie: From the time I was in elementary school, I knew my future career path would involve both science and animals. After I earned a degree in biology, I worked in scientific settings, first in quality control laboratories and finally in a physiology research group at UTMB. I maintained my interest in animals by breeding and raising small and large animals, as well as running an equine boarding stable for several years. Both interests have finally merged in my PAM position.

Judy: Growing up I always had a deep love of animals, and my favorite subject in school was biology. I worked in the pet industry as a groomer and eventually as a manager of pet resorts. I was fortunate enough to find out about UTMB's Animal Resource Center and be hired as an Animal Technician. In my research husbandry career, I was able to use my degree in biology, and I learned about the role research animals play in our daily lives. I worked my way up to a supervisor position, and then I had the opportunity to join the PAM team.

How does each of your backgrounds contribute to your current work?

The phrase 'animal care and use' is encountered repeatedly in animal research. Our backgrounds reflect these two components: Judy's is caring for animals, and Jamie's is using animals in studies. The success of our PAM program is derived from arriving at the common goal of humane animal research through these two pools of experience. We each have a mutual respect for the other's strengths and a willingness to listen to each other's ideas, and we solve problems by building upon each other's knowledge.

Jamie: My favorite way to put researchers at ease is by telling them, "I have been in your shoes longer than I have been in my shoes." I am acutely aware that our work with researchers must benefit the animals, but also the research and the researchers themselves.

Judy: My extensive experience in management and customer service required me to develop my 'people skills'. I use these, combined with my knowledge of research animals, to help our researchers understand the importance of good animal care.

\section{How do you manage to incorporate animal welfare education into your program?}

It was a natural step once we identified that our goal was to help animal researchers in a collegial, educational manner to maintain compliance. Our laboratory reviews consist of protocol discussions and observation of

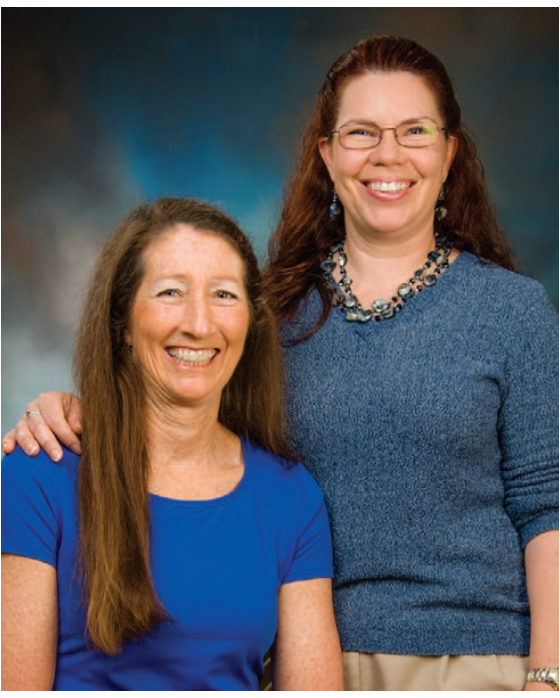

protocol procedures. We encourage an honest exchange of information, allowing researchers to ask procedural and technical questions as well as questions about animal care and compliance. After the observations, we have a debriefing session and, if needed, provide access to training. Once we began conducting laboratory reviews, we realized that there were some common areas where training was needed. As a result, we created educational venues such as a certificate class through our Postdoctoral Affairs office, which includes modules on animal research regulations, the 3 R's, animal numbers justification, literature searches, animal ethics, research advocacy and publishing animal research.

What advice would you give to those developing similar programs at other institutions?

The first step is to determine the main goal of the program. Will it focus just on postapproval monitoring, or will education have a large role? Elucidating the chain of command, and where the authority for the program resides, is especially important when issues with animal welfare and noncompliance are uncovered. Finally, the PAM staff should possess certain personality traits, especially good communication skills as well as flexibility and open-mindedness. The personnel are the driving force behind the program. 